\title{
Philippe Valenti with 27 contributors (Eds.): Tendon transfer for irreparable cuff tear
}

\author{
The GECO Series, Coordinated by Pierre Kehr, Foreword: C. Gerber. Springer, Paris, \\ Berlin, Heidelberg, New York, Hong Kong, London, Milan, Tokyo, 2010; 28 pp; num. \\ figs, color. Figs.; Softcover; € 70,00, ISBN 978-2-8178-0048-6 (Springer Verlag France, \\ Paris, 2011)
}

\section{Pierre Kehr}

Received: 24 November 2011 / Accepted: 26 November 2011/Published online: 7 December 2011

(c) Springer-Verlag 2011

This book can and should be read with pleasure. The reader will find the "real thing" and the "first degree experience" of an author with a great personal involvement in the field of reconstructive shoulder surgery. Each procedure described was performed by the author, and the practical orientation is the rule for this work.

Shoulder surgery is by definition a very anatomic-oriented specialty. The complexity of the structures and the importance of each element in the balance of the algorithm mobility-stability make this discipline very challenging for the specialist involved in shoulder management. Many concerns arise from the variability of the shoulder landmarks, from human morphotypes and from the aging process. The muscles around the shoulder work in synergy, and the deterioration of a part is accompanied by a neighboring reaction that should be understood and addressed when surgery is planned. The biology of the muscular deterioration after a rotator cuff rupture and the variability of the lesions observed make the therapeutic choices difficult. Accurate diagnosis is key for the treatment, and the experience from Philippe Valenti may guide the reader between a latissimus dorsi or a pectoralis major transfer, a deltoid split or a teres major procedure, sometimes, when needed, a combination between a tendon procedure and a prosthetic arthroplasty.
A special attention is granted to surgical details, the techniques are perfectly described, and the anatomy of each repair is outlined. A chapter is dedicated to each muscular transfer in irreparable cuff tears, the latissimus dorsi, the teres major, the pectoralis major, the deltoid flap, but also to techniques of muscular advancement in the retracted tears of the supra- and infraspinatus muscles. A chapter is dedicated to the reverse shoulder arthroplasty associated with a latissimus dorsi and/or teres major transfer for defective external rotation and elevation. This seducing and highly demanding technique is clearly demonstrated and represents one of the best reasons for purchasing the book. The procedure is known and is debated in conferences worldwide, but Philippe Valenti's work shows and explains it clearly, bringing effective solutions for the shoulder specialist in these "not-so rare" occurrences.

Overall, this book may be rated with an A+ and should be in each shoulder specialist personal library. Its reduced volume and intense data gathering recommend this work for a pleasant reading and reference.

Marius M. Scarlat

Hyères

Conflict of interest None.

P. Kehr $(\square)$

Strasbourg, France

e-mail: kehrpier@aol.com 